\title{
The Infrared Multispectral Image Simulation Research Based on 3D Scene
}

\author{
Bo Li ${ }^{1,2,3,4,5}$, Huai-ci Zhao ${ }^{1,3,4}$, Mei Du ${ }^{1,2,3,4}$, Jinfeng Lv ${ }^{1,2,3,4}$ \\ ${ }^{1}$ Department of Optical-Electronics and Information Processing \\ Shenyang Institute of automation, Chinese Academy of Science \\ Shenyang 110016, China \\ 2. University of Chinese Academy of Science \\ Beijing 100049, China; \\ ${ }^{3}$ Key Laboratory of Optical-Electronics Information Processing \\ Chinese Academy of Science \\ Shenyang 110016, China; \\ 4. Key Laboratory of Image Understanding and Computer Vision, Liaoning Province \\ Shenyang 110016, China; \\ ${ }^{5}$ Shenyang Institute of Engineering, Liaoning Province \\ Shenyang 110136,China \\ Email:leebo@sia.cn
}

\begin{abstract}
A method of generating infrared multispectral simulation images by 3D scene is presented. The infrared images at any observation angle of the scene and a data cube of simulation images of specific waveband could be generated by the method. Some experiments have been done in waveband $3 \sim 5 \mu \mathrm{m}$ to generate a data cube involving 401 infrared simulation images in the wavelength step $0.005 \mu \mathrm{m}$, and the experiments results show that the simulation method presented in this paper could generate infrared multispectral images data cube rapidly. A way to reflect the differences while the observation angle changed is mentioned in this paper.
\end{abstract}

Keywords- infrared multispectral simulation, the data cube, self-radiation

\section{INTRODUCTION}

Multi-spectral imaging technology is the new generation of optical-electronics detection technology, and the hot spot in current research and development. The multi-spectral image can make the image information and spectral information together to provide more useful information. The multispectral image can provide not only the 3D scene information as a normal image, but also the spectral radiation information along with wavelength distribution. Multispectral data could form a data cube like figure 1 with strong anti-interference, which can greatly improve the target detection capability and be widely used in military and civil fields ${ }^{[1]}$.

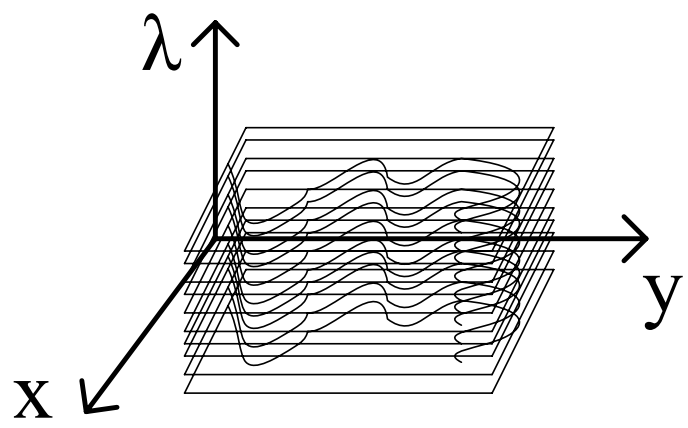

Figure 1. the data cube

Due to technical reasons, most multispectral imaging instruments are controlled by developed countries, and our country only own very few equipment, so it has certain difficulty for multispectral image acquisition. However, related research of multispectral characteristics based on targets and background requires a lot of multispectral images for verification and experiment, therefore how to produce realistic multispectral images by simulation become an urgent need, especially multi-spectral images in the infrared bands.

There are many domestic scholars on the research of IR multispectral image simulation field, but basically focused on the multispectral image generating research from 2D image, such as a $8 \sim 12 \mu \mathrm{m}$ band infrared multispectral image generation method from RGB image proposed by Xu Hong ${ }^{[2]}$ and a 24 hours a day infrared image sequence generation method from RGB image proposed by Chen Shan ${ }^{[3]}$, et al.. These researches are all valuable, but a lot of information was drop away to simplify the research process. Limited by the limitations of $2 \mathrm{D}$ images, it is impossible to generate omnibearing simulation images of the scene. 
In order to solve the problem, this paper presents an infrared band multispectral image generation method based on $3 \mathrm{D}$ scene, by which IR images could be acquired in certain wavelength or specified waveband. The method can not only reserve most of the information of the scene, but also generate multi-angle and comprehensive multispectral images to meet the target image acquisition requirements at any Angle.

\section{The Simulation Method}

In general, the factors affecting the IR thermal radiation image mainly involving the target self-radiation, solar radiation, ground radiation and atmospheric radiation and some other aspects. The thermal radiation model of the target is set up as shown in figure 2, figure of the solid line represents the target itself thermal radiation, and a dotted line represents the target reflected radiation of the incident radiation. Regardless the atmospheric loss of thermal radiation between the target and the sensor, the final target radiation intensity $\left(\mathrm{W} / \mathrm{m}^{2} / \mathrm{sr} / \mu \mathrm{m}\right) L_{m o b j}$ measured by sensor is:

$$
L_{\text {mobj }}=L_{\text {self }}+L_{\text {rsun }}+L_{\text {rsky }}+L_{\text {rground }}
$$

The equation is controlled by 4 parameters:

$L_{\text {self }}:$ the self-radiation intensity of the target

$L_{r s u n}:$ the target reflected radiation intensity of the solar radiation

$L_{r s k y}:$ the target reflected radiation intensity of the sky

$L_{\text {rground }}:$ the target reflected radiation intensity of the ground

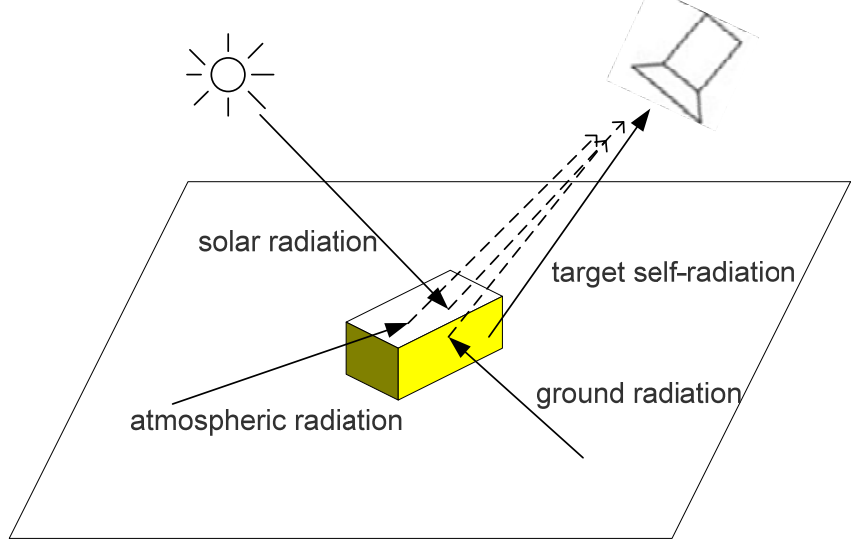

Figure 2. the thermal radiation model of the target

\section{A. the Calculation of the Self-radiation}

The radiation intensity of the target in some waveband like $\left(\lambda_{\mathrm{i}} \sim \lambda_{\mathrm{j}}\right)$ is the integral for all the radiation intensity of the light in every single wavelength in the waveband:

$$
L_{\text {self }}\left(\lambda_{i}, \lambda_{j}\right)=\int_{\lambda_{i}}^{\lambda_{j}} L(\lambda) d \lambda
$$

According to the Planck's law, the irradiance $\left(\mathrm{W} / \mathrm{m}^{2} / \mu \mathrm{m}\right)$ of black body is a function of temperature and wavelength:

$$
M_{b}(\lambda, T)=2 \pi h c^{2} / \lambda^{5}\left(e^{\frac{h c}{\lambda k T}}-1\right)
$$

The radiation intensity of black body is

$L_{b}(\lambda, T)=M_{b}(\lambda, T) / \pi=2 h c^{2} / \lambda^{5}\left(e^{\frac{h c}{\lambda k T}}-1\right)$

Where $\mathrm{h}$ is the Planck constant, and $\mathrm{k}$ is the Boltzmann constant, and $\mathrm{c}$ is the speed of light, and $\mathrm{T}$ is thermodynamic temperature measured in $\mathrm{K}$.

The spectrum radiation intensity of none black body is related to its material:

$$
L(\lambda)=L_{b}(\lambda, T) * \varepsilon(\lambda)
$$

Where $\varepsilon(\lambda)$ is the thermal radiation emissivity of the material.

So the self-radiation intensity of the target in some waveband is:

$$
L_{\text {self }}\left(\lambda_{i}, \lambda_{j}\right)=\int_{\lambda_{i}}^{\lambda_{j}} \varepsilon(\lambda) * 2 h c^{2} / \lambda^{5}\left(e^{\frac{h c}{\lambda k T}}-1\right) d \lambda
$$

The radiation intensity of some target with specific material in one single wavelength or in some waveband could be calculated by equation 6 .

\section{B. the Calculation of the Target Reflected Environment Radiation}

The solar radiation, the ground radiation and the atmospheric radiation totally called environment radiation. The environment radiation reflected by target $E_{\text {ras }}$ is:

$$
\begin{aligned}
L_{\text {ratm }} & =L_{\text {rsun }}+L_{\text {rsky }}+L_{\text {rground }} \\
& =\left(L_{\text {sun }}+L_{\text {sky }}+L_{\text {ground }}\right) * \rho(\lambda)
\end{aligned}
$$

Where $\rho(\lambda)$ is the light reflective in wavelength $\lambda$ of the material of the target.

Because there are too many factors for environment radiation to consider all of them in the calculation, it is necessary to get assistance from professional software as Modtran $^{[4]}$ or Cart.

\section{The Simulation Experiment}

Choose one cooling tower in a power plant in Shenyang area in July as the research target, and build its 3D model shown in figure 3 by Multigen Creator ${ }^{[5]}$, and simulate its radiation characteristics in waveband $3 \sim 5 \mu \mathrm{m}$. Consider the cooling tower as a lambertian emitter in the simulation because it is constructed by relatively rough construction concrete, so the target self-radiation is the same in all directions. 
Set the atmosphere visibility as $23 \mathrm{~km}$, and the sun zenith angle as 30degree, the solar direct radiation calculated by Cart is shown in figure 4.

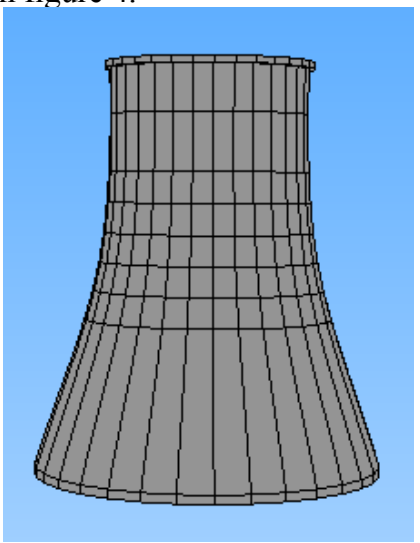

Figure 3. the 3D model of the cooling tower

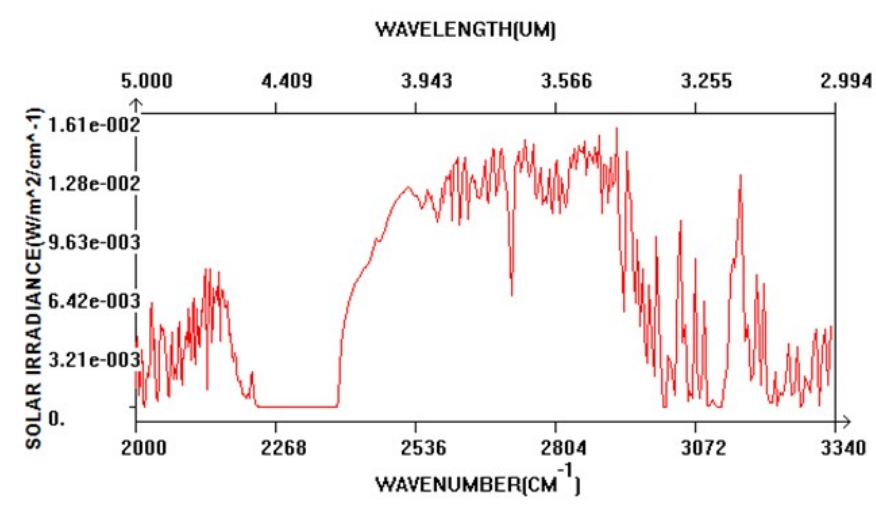

Figure 4. the solar direct radiation in waveband $3 \sim 5 \mu \mathrm{m}$

Set the ground as normal land, and set the temperature as $17^{\circ} \mathrm{C}$, then the background radiation is shown in figure 5 .

\section{WAVELENGTH[UM]}

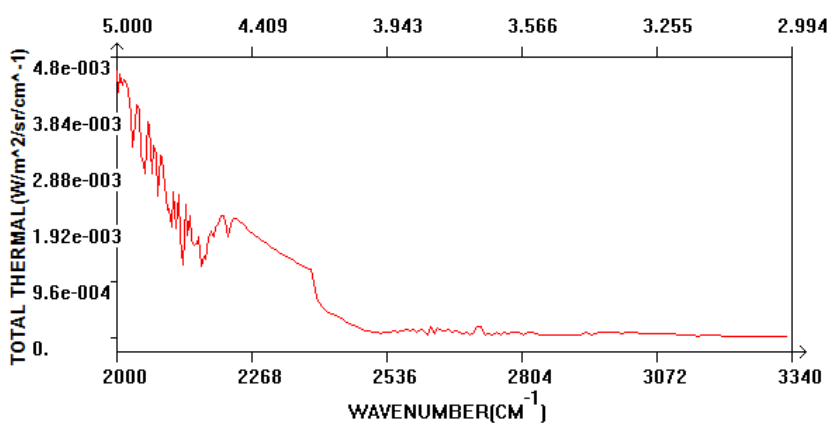

Figure 5. the background radiation in waveband $3 \sim 5 \mu \mathrm{m}$

Set the material of the cooling tower is construction concrete, then acquire the reflectance of the material shown in figure 6 from the ASTER Spectral Library ${ }^{[6]}$ database from JPL(Jet Propulsion Laboratory), US.

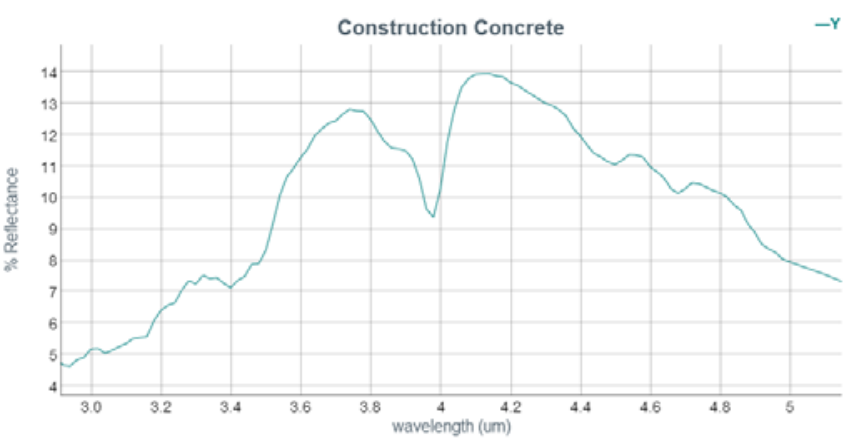

Figure 6. the reflectance of construction concrete in waveband $3 \sim 5 \mu \mathrm{m}$

Set the surface temperature of the cooling tower as $25^{\circ} \mathrm{C}$, regardless the atmospheric loss of thermal radiation between the cooling tower surface and the inherent effects of sensor itself, only considering the radiation characteristics on the surface of the cooling tower, get the data cube of 401 infrared simulation images in waveband $3 \sim 5 \mu \mathrm{m}$ with $\sqcup \lambda=0.005 \mu \mathrm{m}$ by GLSL ${ }^{[7]}$. The simulation images in wavelength $\lambda=3 \mu \mathrm{m}, \lambda=4 \mu \mathrm{m}$ and $\lambda=5 \mu \mathrm{m}$ are shown in figure 7.
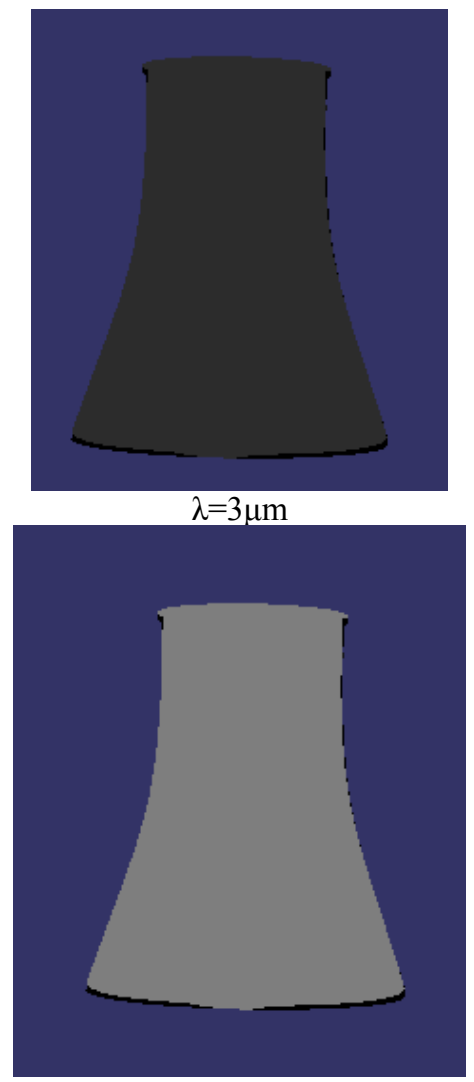

$\lambda=4 \mu \mathrm{m}$ 


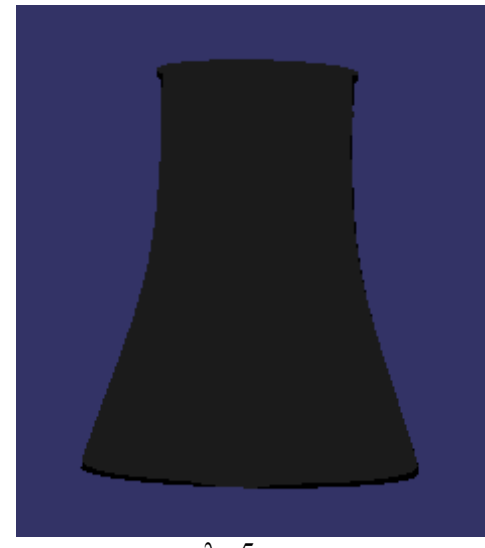

$$
\lambda=5 \mu \mathrm{m}
$$

Figure 7. the simulation images

\section{The Analysis of the Simulation Results}

The simulation results as shown in figure 7 could identify the radiation intensity of the radiation in different wavelength. And with the method mentioned above, the IR simulation images of the scene in any angles could be generated. But no matter how to change the viewing angle, the simulation results don't have a little change. That's because the radiation intensity in this method just has relationship to the incident intensity. The simulation results are acceptable at night time, because the target radiation mainly depends on the self-radiation and the background radiation which don't have obviously directionality. And because of the directionality of solar direct radiation at day time, the simulation results obviously have too much difference with the actual situation.

At present in most of the simulation calculation, the target is approximated to diffuse reflector, which assumes the radiation reflection intensity of the target are equal in all directions. The assumption is reasonable to self-radiation and background radiation, but not proper to solar direct radiation. The BRDF ${ }^{[8][9]}$ (Bidirectional Reflectance Distribution Function) is a function of incident angle, reflected angle and wavelength like shown in figure 8 . It can be used to show the reflection characteristics in different directions of the incident light. BRDF is used here to calculate the target reflection of the solar direct radiation to show the reflection directionality.

\section{SUMMARY}

The simulation method presented in this paper could generate infrared multispectral images cube in any observation angle, any waveband and any step in wavelength. It could improve the accuracy and reusability of the infrared simulation image greatly. The simulation images in different waveband have different radiation characters, which could be used for target recognition.
It is necessary to calculate the specular reflection of the solar direct radiation. The next step of the work is to do some research on a method to calculating the BRDF values in a realtime way.

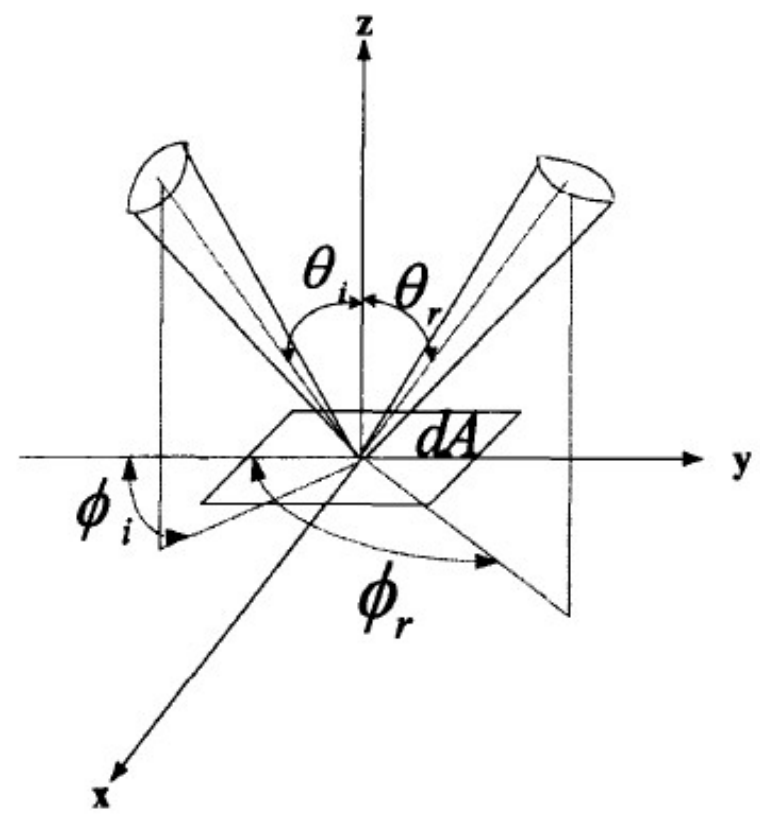

Figure 8. Figure 8: BRDF principle diagram

\section{REFERENCES}

[1] Xu Hong, Wang Xiangjun. Applications of multispectral/hyperspectral imaging technologies in military[J].Infrared and Laser Engineering. 2007,36(1):13-17

[2] Xu Hong, Wang Xiangjun, Liu Feng, et al. Infrared multispectral image simulation based on spectral image in visible bands. Infrared and Laser Engineering. 2009,38(2):201-204

[3] Chen Shan, Sun Jiyin. IR scene simulation based on visual image[J]. Infrared and Laser Engineering. 2009,38(1):23-30

[4] Acharya P K, Berk A,Anderson G P, et al. MODTRAN:Multiple scattering and bi-directional reflectance distribution function upgarads to MODTRAN[J]. Proc of SPIE Optical Spectroscopic Thechniques and Instrumentation for Atmospheric and Space Research III, 1999,3756:354-362

[5] Wang Cheng. Creator Visual Simulation and Modeling Technology[M]. Wuhan: Huazhong university of science and technology press, 2006

[6] Baldridge, A. M., S.J. Hook, C.I. Grove and G. Rivera. The ASTER Spectral Library Version 2.0. Remote Sensing of Environment, 2009,vol 113, pp. 711-715.

[7] Randi J.Rost, Bill Licea-Kane, et al. OpenGL Shading Language 3rd edition[M]. US: Addison-wesley, 2010

[8] Nicodenus F E. Geometrical Considerations and Nomenclature for Reflectance[R]. PB-273439, 1971

[9] Andrew S.Glassner. Principles of Digital Image Synthesis[R]. Morgan-Kaufman, San Francisco, 1995 